\title{
Lo "indígena" en los medios sociales: un análisis bibliométrico desde Scopus
}

The "indigenous" in social media: A bibliometric analysis from Scopus

O "indígena" na mídia social: Uma análise bibliométrica da Scopus

Lebniz DIAZ SUAREZ

Investigador independiente / lebniz.diaz@unmsm.edu.pe

Chasqui. Revista Latinoamericana de Comunicación

N. ${ }^{\circ}$ 146, abril-julio 2021 (Sección Monográfico, pp. 113-140)

ISSN 1390-1079 / e-ISSN 1390-924X

Ecuador: CIESPAL

Recibido: 26-11-202O / Aprobado: 22-02-2021 


\title{
Resumen
}

El objetivo de esta investigación es analizar lo que nos dice la producción científica reunida en la base de datos Scopus sobre la influencia de lo "indígena" en y a través de los medios sociales. Para ello se realizó el análisis bibliométrico de 70 documentos y 52 artículos. Los resultados muestran que el interés de los investigadores por los tópicos que relacionan a lo "indígena" con la nueva ecología mediática ha crecido desde 2013 hasta la fecha de manera progresiva, que Australia cuenta con la mayor producción científica al respecto, y que las implicancias de la participación de lo "indígena" en medios sociales alcanzan lo social, la salud, psicología, tecnología e ingeniería, ciencias ambientales y demás asignaturas. En conclusión, la evidencia científica en Scopus sobre este tema nos dice que hay una evolución positiva de la cuestión indígena que radica en algo más que su visibilidad, pues va evolucionando también con respecto a su ubicuidad y a su glocalidad, manifestación de esto es que avanza del paternalismo hacía el empoderamiento de lo "indígena".

Palabras clave: indígenas; ecología de los medios; medios sociales, análisis bibliométrico, Scopus, medios de comunicación indígenas

\begin{abstract}
The objective of this research is to analyze what the scientific production gathered in the Scopus database tells us about the influence of the "indigenous" in and through social media. For this purpose, the bibliometric analysis of 70 documents and 52 articles was carried out. The results show that the interest of researchers in topics that relate the "indigenous" to the new media ecology has grown from 2013 to date progressively, that Australia has the largest scientific production in this regard, and that the implications of the participation of the "indigenous" people in social media reach the social, health, psychology, technology and engineering, environmental sciences, and so on. In conclusion, the scientific evidence in Scopus tells us that there is a positive evolution of the indigenous issue that lies in more than its visibility; it is also evolving with respect to its ubiquity and its glocality, a manifestation that in this convergent era the indigenous issue is advancing from paternalism towards the empowerment of the "indigenous".
\end{abstract}

Key words: indigenous people; media ecology; social media; bibliometric analysis; Scopus; indigenous media

\section{Resumo}

O objetivo desta pesquisa é analisar o que a produção científica reunida no banco de dados Scopus nos diz sobre a influência de o “indígena” nas e através das mídias 
sociais. Para este fim, foi realizada uma análise bibliométrica de 70 documentos e 52 artigos. Os resultados mostram que o interesse dos pesquisadores em temas que relacionam o "indígena” à nova ecologia da mídia cresceu progressivamente de 2013 até hoje, que a Austrália tem a maior produção científica a este respeito e que as implicações da participação de o "indígena" nas mídias sociais atingem o social, saúde, psicologia, tecnologia e engenharia, ciências ambientais, e assim por diante. Em conclusão, a evidência científica em Scopus sobre tais fatos nos diz que há uma evolução positiva da questão indígena que reside em mais do que sua visibilidade; ela também está evoluindo com respeito a sua ubiqüidade e sua glocalidade, uma manifestação de que nesta era convergente a questão indígena está avançando do paternalismo para o empoderamento de o "indígena".

Palavras-chave: povos indígenas; ecologia das mídias; mídia social; análise bibliométrica; Scopus; mídia indígena

\section{Introducción}

El término indigenous media o “medios de comunicación indígenas" surgió como la expresión política a través de los medios de comunicación frente a la situación adversa de los pueblos indígenas. Mientras iba va cobrando cada vez más presencia como tópico en las bases de datos bibliográficas, se fue convirtiendo en un contemporáneo modo de producción cultural multiplataforma que extiende el deseo de que los pueblos indígenas de todo el planeta, y la etiqueta "indígena", formen parte representativa del mundo (Ginsburg, 1993, 2016; Himpele, 2004).

Se puede notar que cada vez hay más participación de los indígenas, o del tema indígena, en los nuevos medios. No solo en los medios de comunicación convencionales se observa el crecimiento de informaciones y noticias sobre indígenas y sus pueblos, sino también en los medios alternativos, a través de los sitios web, de las aplicaciones para móviles, y especialmente de las redes sociales; es decir, desde la social media o los medios sociales de comunicación (Tsai et al., 2020), alcanzando a posicionarse incluso como tendencia temática en la que es actualmente la más importante y confiable base de datos multidisciplinar existente: Scopus, puesta en marcha por Elsevier y que desde 2004 fue terminando con la supremacía de Web of Science (WoS) en la literatura de ciencia, tecnología, biomedicina y otras disciplinas (Hernández et al., 2016).

A pesar de esta tendencia favorable, el problema que enfrenta la comunidad interesada en la cuestión indígena es que aún no se cuenta con una investigación documental de la producción científica que hay hasta el momento en Scopus sobre el tema de lo indígena en los medios sociales; y como consecuencia de esta realidad, hasta el momento poco se conoce de lo que las referencias en esta famosa base de datos revelan sobre la participación de los indígenas, o del tema indígena, en los nuevos medios. Por tanto, el objetivo de esta investigación es 
analizar lo que nos dice la producción científica reunida en una base de datos tan importante y confiable como Scopus sobre la influencia de lo indígena en y a través de los nuevos medios, los medios sociales.

México con 27.5 millones, Guatemala con 8 millones, Bolivia con 5.5 millones y Perú con 4.4 millones, según Statista (2019), figuran entre los países con mayor número de pobladores indígenas en el mundo, donde se busca extender políticas, tratados, convenios, intervenciones y demás acciones, que alienten, exijan y avalen, el respeto y trato igualitario entre todos los ciudadanos y grupos de ciudadanos de esos países, sea cual sea su cultura, autodefinidas y no autodefinidas como diferentes. En ese ejercicio conjunto de identificación, reconocimiento y revaloración es que descansa el concepto de multiculturalismo (Corona \& Matta, 2020).

En países como México, Guatemala, Bolivia y Perú la multidiversidad es concebida generalmente como riqueza y la data sustenta su alta rentabilidad y demás beneficios que surgen al apostar por lo plural y por los conocimientos ancestrales que provienen de ella en ámbitos ya consolidados; por ejemplo, pedagogía en México (Rosado \& Francisco, 2017), minería en Ecuador (García, 2014), salud en Perú (Cárdenas, Pesantes, \& Rodríguez, 2017), gastronomía en México y Perú (Corona \& Matta, 2020). Así, el consenso especialista no solo diagnostica que los problemas de interculturalidad, que afectan a lo indígena y a los indígenas, proceden de la falta de acceso al poder, sino que la comunicación y la interacción en todas las áreas vinculadas a la comunicación permiten ese acceso (Scheinsohn, 2014); en este proceso, los medios sociales se han tornado cruciales.

Vigilar lo que hasta el momento se dice académicamente sobre lo que hacen los indígenas en las nuevas plataformas permite tener mejor panorama de esta participación mediática, su tendencia hacia el desarrollo o entorpecimiento del progreso de los pueblos indígenas, y las dimensiones que son favorecidas y desfavorecidas mientras consumen y producen relatos relacionados a sus comunidades, costumbres e intereses. El indígena, pues, también participa de las nuevas comunicaciones, es un generador de contenidos, y por ello cada vez se realizan más estudios sobre la participación de los indígenas en medios sociales y el impacto para sus pueblos.

Entonces, es pertinente seguir analizando datos, trabajos científicos y documentación confiable relacionada a la cuestión indígena y a la vigente ecología mediática indígena, y acercarnos más a la influencia internacional de lo indígena a través de los nuevos medios, gestionada por ellos, con ellos o para ellos; como también acercarnos más al beneficio mundial del reconocimiento de sus derechos. 


\section{Marco teórico}

\subsection{La cuestión "indígena"}

Con los estudios etimológicos de Varrón, Virgilio, Tito Livio, Estacio, Plinio y otros más, se sabe que el término "indígena” proviene del latín indigěná, que a su vez está conformado por el prefijo (o adverbio) latino inde (indu-, indi-) que remite a "en el interior (de allí)" y de gen, que es la raíz de los verbos gigněre, que significa "engendrar, parir" y generāre, que también significa "engendrar". Cristóbal Colón llamó "indios" a los nacidos en América al creer que había llegado a Las Indias. Aún se asocia, equivocadamente, la palabra "indígena" con el término "indio". El término "indio" viene del latín india, que a su vez tiene como raíz la voz persa hindu y este del sanscrito sindhu, que significa "río, corriente de agua”. En la edición 23 del Diccionario de la Lengua Española, actualización 2019 (Real Academia Española, 2001), la única acepción de la palabra "indígena" es "originario del país de que se trata". En el DRAE, la palabra "originario", del latín originarius, tiene dos acepciones; la primera: "que da origen a alguien o algo", y la segunda: "que trae su origen de algún lugar, persona o cosa".

La propuesta de definir "indígena" con relación al "nacimiento" y el "lugar de nacimiento" de una persona puede generar más confusiones sobre qué y quién es indígena. Los humanos somos nativos u originarios de alguna tierra y no por eso todos somos indígenas. Las referencias mencionadas ahondan más y más en el origen de las personas y de sus expresiones, para definirlas como "indígenas", rebuscando en la ascendencia hasta encontrar al antepasado menos intercultural. Así, a lo indígena se le exige ser algo fijo, no dinámico, estar lo más aislado posible de lo urbano; por ejemplo, cuando un habitante de una cultura distinta tiene más contacto con la urbanización se le cree menos indígena. Para el imaginario colectivo, algo o alguien es más indígena cuando menos intercultural es; pero paradójicamente el término "indígena” nació del intercambio cultural, sorprende que aún en la actualidad esté distanciado de lo intercultural para su conceptualización.

Las ONU no encasilla la definición de "indígena", no obstante, encontró en los estudios de Martínez Cobo (1981) una base de trabajo y un marco intelectual para y con las comunidades, poblaciones y naciones indígenas que incluye sus propios derechos a definir qué y quién es indígena. Entonces, la primera definición de trabajo corresponde a "poblaciones indígenas":

Aquellas que, teniendo una continuidad histórica con las sociedades invasoras y precoloniales que se desarrollaron en sus territorios, se consideran a sí mismas distintas de otros sectores de las sociedades que actualmente prevalecen en dichos territorios o en parte de estos. Actualmente, ellas forman sectores no dominantes de la sociedad y están decididas a preservar, desarrollar y transmitir a las generaciones futuras sus territorios ancestrales y su identidad étnica como base 
de su existencia continuada como pueblos, de acuerdo con sus propios patrones culturales, instituciones sociales y sistemas jurídicos. (Naciones Unidas, s.f.).

La continuidad histórica a la que se refiere remite a un tiempo prolongado hasta el presente, y que alcanza, en resumen, uno o dos de estos factores: 1. Ocupación total o en parte de tierras ancestrales; 2. Antepasados compartidos con anteriores ocupantes de esas tierras; 3. Manifestaciones culturales (religión, vestimenta, medios de vida, música, y otros); 4. Lengua, sea usada como única, como lengua materna, principal, preferida o habitual; 5. Residencia en ciertas partes del país o regiones del mundo; y 6. Otros aspectos relevantes.

De esta forma, la segunda, y la que más nos interesa, es la definición de trabajo para el término "indígena":

Una persona indígena es aquella que pertenece a estas poblaciones indígenas que se autoidentifican como indígenas (conciencia grupal) y es reconocida y aceptada por estas poblaciones como uno de sus miembros (aceptación por el grupo). Esto permite a estas comunidades conservar el derecho soberano y el poder de decidir quién pertenece a ellas, sin interferencias externas. (Naciones Unidas, s.f.)

Se sabe que en el mundo hay alrededor de 370 millones de indígenas en más de 90 países, constituyendo $15 \%$ de la población mundial que vive en pobreza extrema (Banco Mundial, 2018). Los pueblos indígenas siguen enfrentando situaciones de marginación, de pobreza extrema y otras violaciones de derechos humanos (Unesco, s.f.); por tal razón, se han elaborado los convenios internacionales enmarcados en los derechos de los pueblos minoritarios, como el 169 (OIT, 2014).

\subsection{La ecología de los medios sociales indígenas}

A medida que un mayor número de personas tiene acceso a la tecnología, sus necesidades y objetivos de comunicación también se diversifican; desde que populares redes sociales como Facebook, Youtube $y$ Twitter se han integrado a los teléfonos, surgen nuevas herramientas y tecnologías que los usuarios usan para acceder y compartir información, extendiéndose así poblaciones cada vez más heterogéneas que convergen en múltiples plataformas mientras satisfacen sus necesidades comunicativas. Dichas poblaciones son cada vez más conscientes de esta nueva ecología mediática y por ello fortalecen cada vez más sus posibilidades de comunicación e información a través de todas las diversas herramientas disponibles y no solo de un único canal concreto y restringido (Zhao, 2016).

Los medios de comunicación social, la social media, conocidos simplemente como "medios sociales", son las tecnologías de comunicación no tradicionales, alternativas a los medios de comunicación de masas (mass media), utilizados como sistemas de conocimiento e interacción social a través de contenidos 
producidos y reproducidos por sus usuarios, ya sea sobre un interés común o una realidad social de la que se demanda información. En manos de determinados actores, los medios sociales se muestran como plataformas que sirven como herramientas para perseguir objetivos específicos (Roca, 2007; Leonardi et al., 2013; Poell, 2014).

La Ecología de los Medios es un postulado de las comunicaciones que se viste para algunos como teoría en sí y para otros como metadisciplina; lo categórico es que su objeto de estudio es el impacto social producido por las tecnologías y medios de comunicación (Islas, 2009; 2015). Cuenta con dos perspectivas: los medios como ambientes, donde conviven los seres humanos, y los medios como especies, donde conviven las tecnologías entre ellas mismas (Hidalgo, 2016, p. 182).

Esta teoría comunicacional, cuyos padres fundadores son Marshall McLuhan y Neil Postman (Scolari, 2015), es la más vigente y como tal tiene influencia en variados aspectos vinculados al desarrollo mediático: desde el entretenimiento, marketing, periodismo, hasta la comunicación política. Todos estos campos, y otros más, se caracterizan actualmente por su expansión transmedial y convergente, donde la comunicación no funciona aisladamente sino entrelazando los medios tradicionales con todo el nuevo ecosistema digital, lo que le otorga potencial político (Riorda, 2017).

La nueva ecología mediática ha evolucionado en esa actual convergencia que dinámicamente reconfigura la forma de relatar, de contar historias, y que cede la construcción y reconstrucción de mundos narrativos desde la colaboración cooperativa de aquellos interesados en lo que se narra en los nuevos medios de comunicación (Jenkins et al., 2015; Scolari et al., 2018). Si bien, frente al surgimiento de prosumidores (productores + consumidores) en distintas latitudes, orígenes, geografías y etnias, actualmente sobresalen enunciados tan optimistas, como los de la Ecología de los Medios (McLuhan, 1962, 1964; Postman, 1970, 1988), y de ellas se extienden las de la Cultura de la Convergencia (Jenkins, 2006), y Narrativas Transmedia (Jenkins, 2003; Scolari, 2013), pues también con optimismo se va reconociendo al indígena como otro miembro más de las interfaces globales, como los medios sociales, donde sus asuntos y los asuntos del mundo convergen a manera de relatos.

Recuérdese el surgimiento y evolución de la indigenous media, o de los “medios de comunicación indígenas", que revela cómo se va afianzando cada vez más la cuestión "indígena" en la agenda global, y que ya dejó de solo advertir la situación desfavorable de las poblaciones indígenas para ahora promover además sus riquezas culturales y alcanzar más vigencia representativa en el mundo (Ginsburg 1993, 2016; Himpele 2004). Por tanto, de acuerdo al "derecho de los pueblos indígenas a mantener y fortalecer sus culturas, formas de vida e instituciones propias, y su derecho a participar de manera efectiva en las decisiones que les afectan" (OIT, 2014), es necesario enfatizar que cada vez los mismos pobladores indígenas y los temas indígenas van participando más 
de la nueva ecología mediática y así van haciendo circular más narrativas que corresponden a sus culturas, necesidades, obstáculos y avances.

\section{Metodología}

El análisis bibliométrico es una técnica científica cuantitativa que consiste en la búsqueda y revisión sistemática, y también replicable, de información transparente y objetiva, a manera de meta-datos, sobre una temática específica dentro de la producción científica, para conocer sus características, fortalezas, debilidades y evolución en los últimos años; lo cual resulta de gran interés para los gestores de las revistas académicas y para los investigadores que publican en ellas (Ariza \& Quevedo, 2012; Pérez, 2019; Wang \& Ngai, 2020).

La comunidad científica concuerda en que Scopus es uno de los mejores repositorios de la visibilidad del conocimiento humano y la mayor base de datos multidisciplinar existente de citas y resúmenes de bibliografía de revistas científicas, libros y actas de conferencias revisadas por pares. Elsevier lo puso en marcha desde 2004 para que los investigadores a escala mundial puedan visualizar, acceder, seguir y analizar la literatura sobre ciencia, tecnología, medicina, ciencias sociales, artes y humanidades; en el año 2020, esta base de datos ya cuenta con 24.600 títulos de revistas científicas, 75 millones de registros (Hernández et al., 2016; Chávez, 2020; Elsevier, 2021).

Se sabe que la difusión del conocimiento es esencial para el avance de la ciencia; frente a ello, el uso de indicadores bibliométricos presenta múltiples ventajas para evaluar la producción científica, explorando los artículos influyentes, hallando tendencias de popularidad, o rastreando el surgimiento y evolución de grupos y metodologías de investigación. Scopus muestra un sistema de búsqueda importante para identificar las revistas científicas donde incluye los indicadores bibliométricos utilizados en la evaluación del factor de impacto, que permite comparar la importancia de una publicación respecto a otras pertenecientes a una misma área de conocimiento, que se mide tomando en cuenta la cantidad de fascísculos por año, el número de citas bibliográficas, las referencias bibliográficas, el número de descargas por base de datos y área geográfica (Velasco et al., citado por Ariza \& Quevedo, 2012; Chávez, 2020; Wang \& Ngai, 2020). Estos indicadores se atienden también en este análisis bibliométrico frente a la necesidad investigativa de rastrear objetivamente las publicaciones académicas, hallar las investigaciones más influyentes, identificar categorías y proporcionar ideas para futuros trabajos sobre el tema de lo indígena en y a través de los medios sociales.

Para alcanzar el objetivo de esta investigación, se realizó el análisis por recuento de frecuencias y cruce de variables de los documentos y, principalmente, de los artículos científicos hallados en la base de datos de Scopus, que abordan el tema de lo indígena desde las nuevas comunicaciones. Esto se organizó a manera de meta-datos, que sintetizan las evidencias más 
importantes y accesibles a través de una búsqueda, tratado, plan de trabajo y criterios totalmente esclarecidos y que pueden ser replicadas por otros para llegar a las mismas conclusiones (Universitat Politècnica de València, 2015).

La búsqueda de los documentos se realizó en primer momento de manera exploratoria, acudiendo a combinaciones de palabras claves, en castellano y en inglés, que delimiten cada vez mejor las referencias y generen mayor calidad de resultados. De esta manera, la exploración en Scopus permitió fijar como lengua de búsqueda al inglés, debido a la mayor cantidad de documentos resultantes -incluso las referencias en castellano y otras lenguas incluyen una traducción al inglés- y así encontrar los operadores, etiquetas y combinaciones más eficientes. Con estos criterios, se eligió finalmente para la búsqueda avanzada de documentos el uso de la siguiente fórmula booleana:

( TITLE ( "indigenous") AND TITLE-ABS-KEY ( "social media" ) AND ALL ( "impact” OR "influence" OR "political" OR "rights" ) ).

La ecuación generó 70 documentos sobre la participación indígena en medios sociales y el impacto de esa participación en sus pueblos, a los que se les realizó un análisis bibliométrico (tablas y gráficas estadísticas). De ellos, se limitaron los resultados bajo el criterio de reunir solo artículos y se obtuvieron definitivamente 52, organizados en una hoja de cálculo de Microsoft Excel con este protocolo de análisis: ID, título de documento, autor(es), año, fuente, número de citas, resumen (en inglés), traducción del resumen (castellano), temas, percentil de prominencia, palabras clave, DOI, lengua original, número de referencias, referencia bibliográfica (APA 6ta).

Tabla 1. Instrumento de análisis

\begin{tabular}{|l|l|l|}
\hline ID & número de citas & palabras clave \\
\hline título de documento & resumen (en inglés) & DOI \\
\hline autor(es) & traducción del resumen (castellano) & lengua original \\
\hline año & temas & número de referencias \\
\hline fuente & percentil de prominencia & referencia bibliográfica (APA 6ta) \\
\hline
\end{tabular}

Fuente: Elaboración propia

\section{Resultados}

Con la revisión sistemática hallamos coincidencias informativas que ayudan a conocer más sobre lo indígena y la influencia que estos pueblos pueden alcanzar en el mundo con las narrativas indígenas producidas y reproducidas a través de las redes, y resumimos la documentación de artículos en Scopus sobre el tema. De esta manera, presentamos los resultados más pertinentes a continuación. 
Tabla 2. Top 5 de artículos sobre influencia y participación indígena en medios sociales por número de citas

\begin{tabular}{|l|l|l|l|l|l|}
\hline ID & Título de documento & Autor & Año & Fuente & Citas \\
\hline 45 & $\begin{array}{l}\text { Indigenous rights, performativity } \\
\text { and protest }\end{array}$ & $\begin{array}{l}\text { Hanna, P., } \\
\text { Langdon, E.J., \& } \\
\text { Vanclay, F. }\end{array}$ & 2016 & $\begin{array}{l}\text { Land Use Policy } \\
50, \text { pp. 490-506 }\end{array}$ & 30 \\
\hline 43 & $\begin{array}{l}\text { Indigenous voices and mediatized } \\
\text { policy-making in the digital age }\end{array}$ & $\begin{array}{l}\text { Dreher, T., } \\
\text { McCallum, K., \& } \\
\text { Waller, L. }\end{array}$ & 2016 & $\begin{array}{l}\text { Information } \\
\text { Communication } \\
\text { and Society } \\
19(1), \text { pp. 23-39 }\end{array}$ & 21 \\
\hline 31 & $\begin{array}{l}\text { Evidence for a comprehensive } \\
\text { approach to Aboriginal tobacco } \\
\text { control to maintain the decline in } \\
\text { smoking: An overview of reviews } \\
\text { among Indigenous peoples }\end{array}$ & $\begin{array}{l}\text { Chamberlain, } \\
\text { C., Perlen, S., } \\
\text { Brennan, S., (..), } \\
\text { Wilson, A., \& } \\
\text { Eades, S. }\end{array}$ & 2017 & $\begin{array}{l}\text { Systematic } \\
\text { Reviews } \\
6(1), 135\end{array}$ & 18 \\
\hline 48 & $\begin{array}{l}\text { The listening key: Unlocking the } \\
\text { democratic potential of indigenous } \\
\text { participatory media }\end{array}$ & $\begin{array}{l}\text { Waller, L., } \\
\text { Dreher, T., \& } \\
\text { McCallum, K. }\end{array}$ & 2015 & $\begin{array}{l}\text { Media } \\
\text { International } \\
\text { Australia } \\
(154), \text { pp. 57-66 }\end{array}$ & 17 \\
\hline \multirow{5}{*}{51} & $\begin{array}{l}\text { The use of social media } \\
\text { technologies to create, preserve, } \\
\text { and disseminate indigenous } \\
\text { knowledge and skills to } \\
\text { communities in East Africa }\end{array}$ & $\begin{array}{l}\text { Owiny, S.A., } \\
\text { Mehta, K., \& } \\
\text { Maretzki, A.N. }\end{array}$ & 2014 & $\begin{array}{l}\text { International } \\
\text { Journal of } \\
\text { Communication } \\
8(1), \text { pp. } \\
\text { 234-247 }\end{array}$ & 17 \\
\hline
\end{tabular}

Fuente: Scopus

Acerca de la distribución de documentos por año, en la Tabla 3 y en el Gráfico 1, presentamos los 70 documentos, tomando como referencia el número de publicaciones hechas por año. A través de los datos se puede conocer que es a partir del año 2013 que se abre en Scopus la documentación sobre influencia y participación indígena en los nuevos medios, y que el interés por abordar cada vez más investigaciones relacionadas a estos temas fueron creciendo en número año tras año. Si solo hasta la fecha, septiembre de 2020, hay 14 documentos que ya ingresaron a esta base de datos, entendemos que hasta fin de año aún más documentos podrían sumarse. Sin embargo, por el momento ha sido el 2018, con 15 documentos, el año de mayor producción.

Tabla 3. Distribución de documentos por año

\begin{tabular}{|c|c|c|c|}
\hline Año & Documentos & Año & Documentos \\
\hline 2020 & 14 & 2016 & 12 \\
\hline 2019 & 10 & 2015 & 4 \\
\hline 2018 & 15 & 2014 & 3 \\
\hline 2017 & 11 & 2013 & 1 \\
\hline
\end{tabular}

Fuente: Scopus 
Gráfico 1. Documentos por año

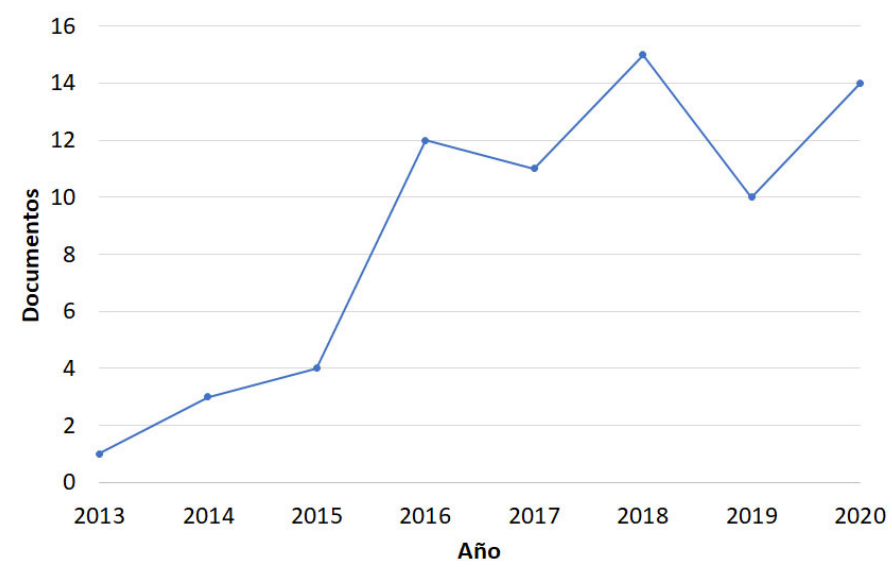

Fuente: Scopus

En la distribución de tipo de documentos por número encontramos mayoritariamente artículos científicos sobre lo indígena en los medios sociales. Según la Tabla 4 son 52 artículos publicados en Scopus, representando el 74,3\% de la totalidad de documentos (ver Gráfico 2) y solo hay 2 libros sobre el tema: Creating dialogues: Indigenous perceptions and changing forms of leadership in Amazonia (Veber \& Virtanen, 2017) y The geography of names: Indigenous to post-foundational (Eades, 2016).

Tabla 4. Distribución de tipo de documentos por número

\begin{tabular}{|l|c|}
\hline \multicolumn{1}{|c|}{ Tipo de documento } & Documentos \\
\hline Artículo & 52 \\
\hline Revista & 8 \\
\hline Capítulo de libro & 4 \\
\hline Documento de conferencia & 3 \\
\hline Libro & 2 \\
\hline Carta & 1 \\
\hline
\end{tabular}

Fuente: Scopus 
Gráfico 2. Tipo de documentos por número

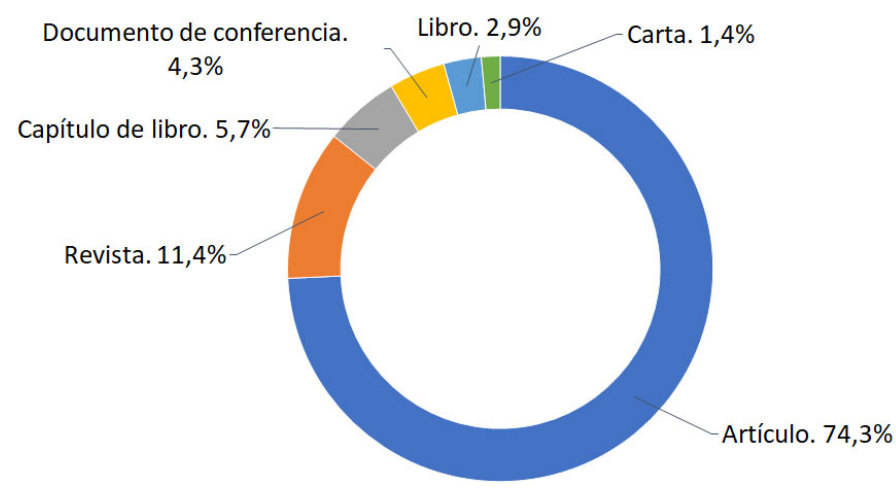

Fuente: Scopus

Según La distribución de documentos por patrocinador financiador (Tabla 4. y Gráfico 3) existen 3 patrocinadores liderando la financiación de producción científica relacionada a lo indígena en medios sociales; estas son el Consejo Australiano de Investigación, Consejo Nacional de Salud e Investigación Médica de Australia) y el Consejo de Investigación de Ciencias Sociales y Humanidades de Canadá, cada una con 3 documentos.

Tabla 4. Distribución de documentos por patrocinador

\begin{tabular}{|l|c|}
\hline \multicolumn{1}{|c|}{ Patrocinador financiero } & Documentos \\
\hline Australian Research Council & 3 \\
\hline National Health and Medical Research Council & 3 \\
\hline Social Sciences and Humanities Research Council of Canada & 3 \\
\hline Economic and Social Research Council & 2 \\
\hline Alfred Kordelinin Säätiö & 1 \\
\hline Arts and Humanities Research Council & 1 \\
\hline AstraZeneca Canada & 1 \\
\hline Conselho Nacional de Desenvolvimento Científico e Tecnológico & 1 \\
\hline Coordenação de Aperfeiçoamento de Pessoal de Nivel Superior & 1 \\
\hline Fundação de Amparo à Pesquisa do Estado do Amazonas & 1 \\
\hline Medical Research Council & 1 \\
\hline Ministry of Business, Innovation and Employment & 1 \\
\hline Norges Forskningsråd & 1 \\
\hline University of Manitoba & 1 \\
\hline University of Windsor & 1 \\
\hline
\end{tabular}

Fuente: Scopus 
Gráfico 3. Documentos por patrocinador

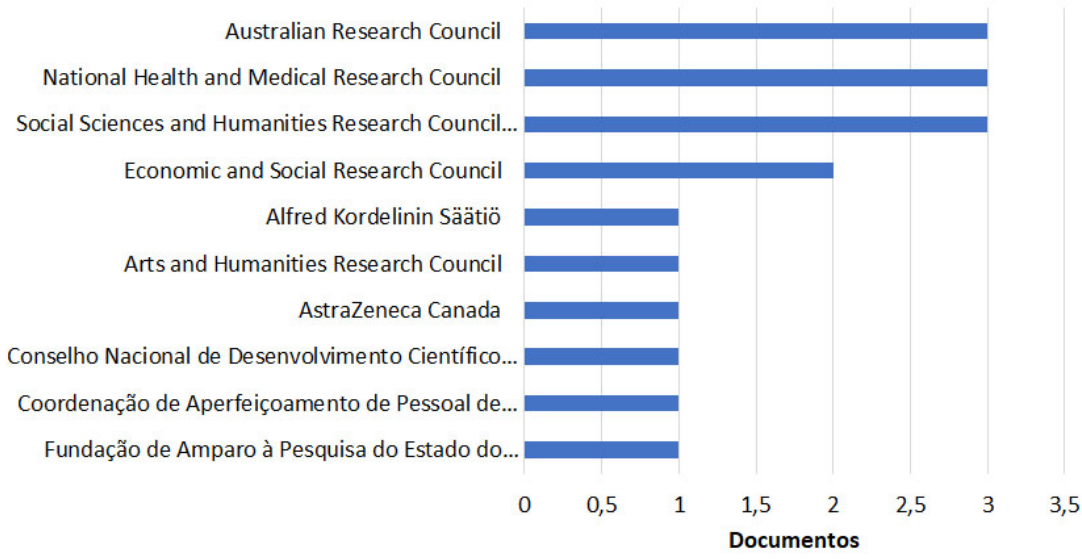

Fuente: Scopus

El recuento de la documentación que hay en la base de datos de Scopus sobre el tema tiene como área temática más interesada a las Ciencias Sociales, con 51 documentos y 45,9\% del total de los documentos publicados, según la Tabla 5 y Gráfico 4. Así, según lo recolectado en esta distribución de documentos por área temática, tenemos que, alcanzando el 12,6\% del total documentado, la siguiente área temática más interesada es la de Arte y Humanidades con 14 documentos, y muy cerca, cada una con el 10\% de toda la documentación al respecto, están la Medicina (11 documentos, 9,9\%) y las Ciencias de la Computación (10 documentos, $9 \%)$.

Tabla 5. Distribución de documentos por área temática

\begin{tabular}{|l|c|l|c|}
\hline \multicolumn{1}{|c|}{ Área temática } & Documentos & \multicolumn{1}{|c|}{ Área temática } & Documentos \\
\hline Ciencias Sociales & 51 & Ingeniería & 2 \\
\hline Artes y Humanidades & 14 & Multidisciplinario & 2 \\
\hline Medicina & 11 & Psicología & 2 \\
\hline Ciencia de la computación & 10 & Ciencias Agrícolas y Biológicas & 1 \\
\hline Negocios, Gestión y Contabilidad & 7 & Ciencias de la Tierra y del Planeta & 1 \\
\hline Ciencias de la decisión & 5 & Economía, Econometría y Finanzas & 1 \\
\hline La ciencia ambiental & 3 & Enfermería & 1 \\
\hline
\end{tabular}

Fuente: Scopus 
Gráfico 4. Documentos por área temática

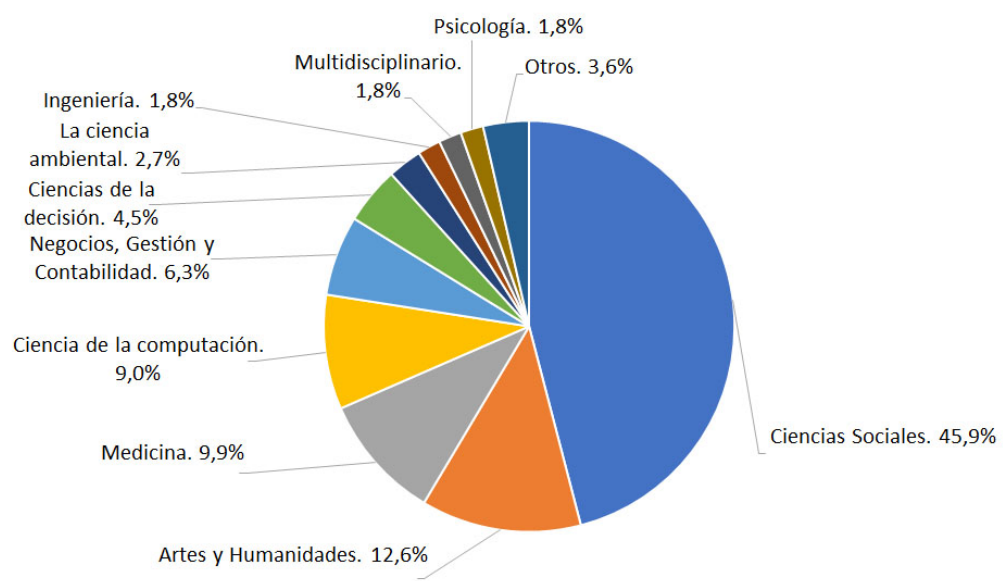

Fuente: Scopus

En la distribución de documentos por país/territorio, como ya podíamos notar en la clasificación de documentos por patrocinador, aquella superioridad de las organizaciones australianas como patrocinadores y financistas de documentos, aquí ya también queda revelado que en Australia recae la mayor cantidad de producción científica, 22 documentos, relacionada al uso que hacen los indígenas de los nuevos medios. Y los otros países oceánicos en sumarse a estos estudios son Nueva Zelanda (con 4 documentos) y Fiyi (con 1 documento).

Si seguimos observando la Tabla 6 y el Gráfico 5 veremos que, según Scopus, los territorios donde más autores han tomado interés por las implicancias de la actual ecología mediática de los indígenas son Canadá y Estados Unidos, con 16 documentos y 15 documentos, respectivamente, y a ellos se les suma México que está ingresando a la base de datos con 1 publicación al respecto. Mientras que en el caso sudamericano, encontramos que revistas de Brasil y Ecuador, con 1 documento cada uno, son las únicas de la región en lograr figurar en Scopus con sus trabajos científicos que relacionan las poblaciones indígenas con los medios sociales. Estas publicaciones son: Indigenous rights, performativity and protest (Hanna, Langdon, \& Vanclay, 2016), en Brasil, y Use of information and communications technologies by Indigenous civil society organizations in Ecuador (Lupien \& Chiriboga, 2019), en Ecuador. En territorio africano encontramos 3: Nigeria (2) y Sudáfrica (1); en Asia van 2: India (1) y Filipinas (1); y en Europa suman 14: Reino Unido (7), Finlandia (3), Dinamarca (1), Holanda (1), Noruega (1) y Suecia (1). 
Tabla 6. Distribución de documentos por país/territorio

\begin{tabular}{|l|c|l|c|}
\hline \multicolumn{1}{|c|}{ País/territorio } & Documentos & País/territorio & Documentos \\
\hline Australia & 22 & Fiji & 1 \\
\hline Canadá & 16 & India & 1 \\
\hline Estados Unidos & 15 & México & 1 \\
\hline Reino Unido & 7 & Países Bajos & 1 \\
\hline Nueva Zelanda & 4 & Noruega & 1 \\
\hline Finlandia & 3 & Filipinas & 1 \\
\hline Nigeria & 2 & Sudáfrica & 1 \\
\hline Brasil & 1 & Suecia & 1 \\
\hline Dinamarca & 1 & Sin definir & 3 \\
\hline Ecuador & 1 & & \\
\hline
\end{tabular}

Fuente: Scopus

Gráfico 5. Documentos por país/territorio

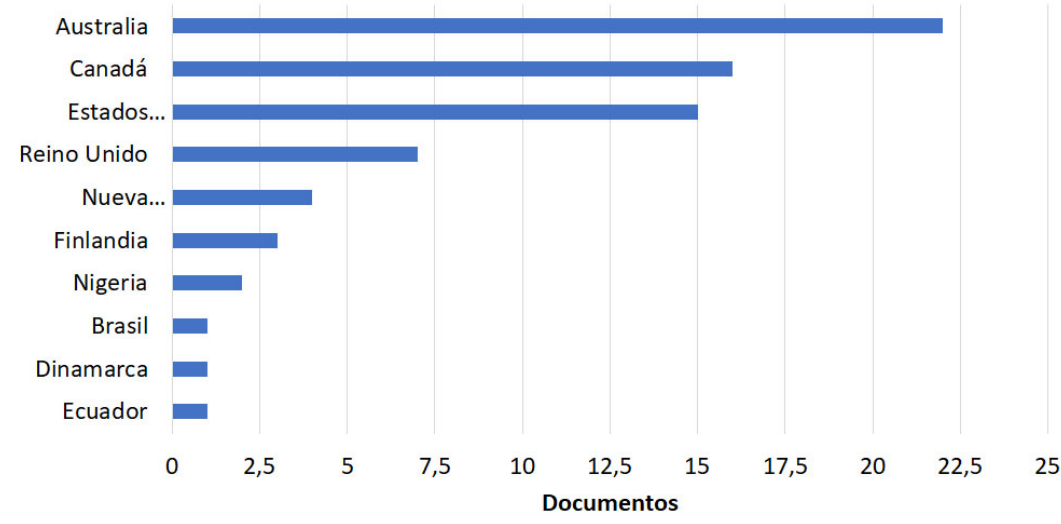

Fuente: Scopus

El análisis de la distribución de documentos por afiliación nos da la cantidad de 104 universidades en el mundo que figuran como instituciones educativas afiliadas a documentos que tratan el caso de la influencia de la participación indígena de los medios sociales. Cabe destacar la importancia de producción científica australiana al respecto. La mayor parte de estas 104, universidades se encuentran ubicadas en Australia, la Universidad de Canberra es la que más documentos afiliados presenta (6); en el top 10, las 6 primeras universidades con mayor número de documentos en Scopus por afiliación son australianas, 
sumando solo esas universidades la cantidad de 22. En cuanto a las universidades latinoamericanas que figuran en la lista de documentos por afiliación están la Universidade Federal de Santa Catarina (Brasil), la Benemerita Universidad Autónoma de Puebla (México) y la Universidad Técnica del Norte (Ecuador), todas ellas con la cantidad de 1 documento.

Tabla 7. Distribución de documentos por afiliación (top 10)

\begin{tabular}{|l|c|}
\hline \multicolumn{1}{|c|}{ Afiliación } & Documentos \\
\hline Universidad de Canberra & 6 \\
\hline La Universidad Nacional de Australia & 4 \\
\hline Universidad de Wollongong & 3 \\
\hline La Universidad Deakin & 3 \\
\hline Universidad Macquarie & 3 \\
\hline La Universidad de Sydney & 3 \\
\hline Universidad de Calgary & 2 \\
\hline Universidad McGill & 2 \\
\hline Universidad Estatal de Arizona & 2 \\
\hline Universidad de Auckland & 2 \\
\hline
\end{tabular}

Fuente: Scopus

Gráfico 6. Documentos por afiliación

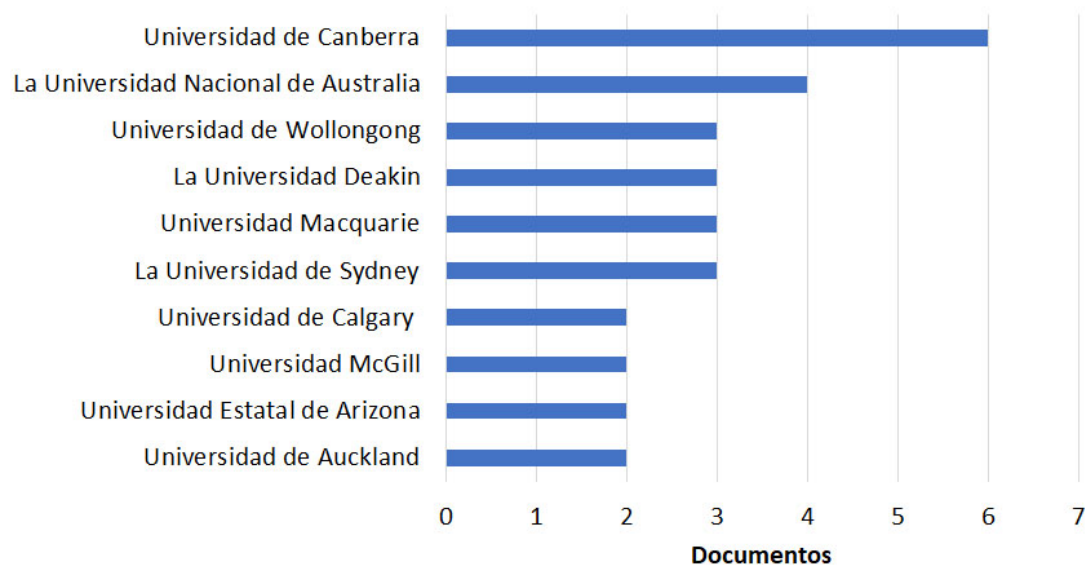

\section{Fuente: Scopus}


El recuento de la distribución de documentos por autor revela que en Scopus hay 160 autores hasta el momento que han generado documentación sobre la implicancia de la participación de los indígenas en medios sociales. Como podemos ver en la Tabla 8 y Gráfico 7, tenemos a Dreher, T. como el autor con más publicaciones al respecto en esta base de datos, con 4 documentos, a este le siguen Carlson, B., McCallum, K., Waller, L., con 3 publicaciones cada uno. Tanto Dreher, Carlson, McCallum y Waller han realizado participaciones conjuntas en sus trabajos investigativos que relacionan a los indígenas australianos con los nuevos medios.

Tabla 8. Distribución de documentos por autor (top 10)

\begin{tabular}{|l|c|l|c|}
\hline \multicolumn{1}{|c|}{ Nombre de autor } & Documentos & Nombre de autor & Documentos \\
\hline Dreher, $\mathrm{T}$. & 4 & Frazer, R. & 2 \\
\hline Carlson, B. & 3 & Kral, I. & 2 \\
\hline McCallum, K. & 3 & Lupien, P. & 2 \\
\hline Waller, L. & 3 & Adedeji, A. & 1 \\
\hline Duarte, M. & 2 & Aguilera, R. & 1 \\
\hline
\end{tabular}

Fuente: Scopus

Gráfico 7. Documentos por autor

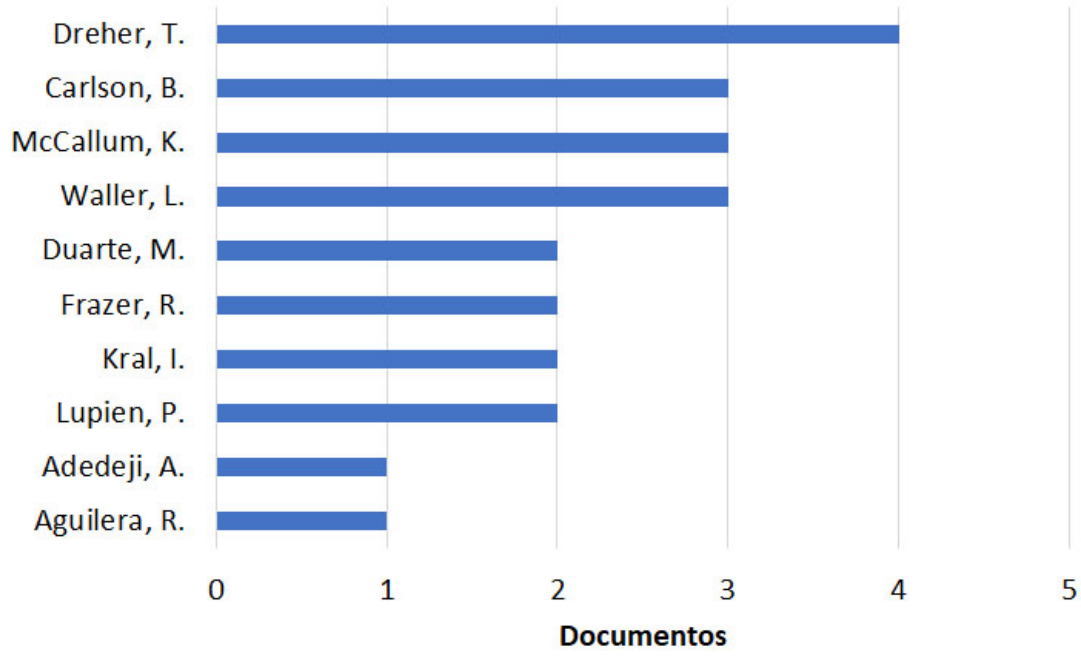

Fuente: Scopus

A través de la distribución de documentos por año y fuente, el análisis nos revela que son 48 revistas indizadas a Scopus las que han publicado hasta 
el momento documentos relacionados al uso de los nuevos medios por los indígenas del mundo. A través de la Tabla 9 y Gráfico 8 podemos reconocer el ranking de las 5 primeras revistas que han tomado en cuenta estos temas. Estos datos estadísticos muestran que la revista Information Communication And Society es junto a la revista Media International Australia los medios científicos que más documentos tienen por año. También muestra que hay un liderazgo de las revistas australianas en el tratado indígena de los medios sociales, y esta revista Media International Australia es la más constante y progresiva productora de contenido académico al respecto, avanzando desde el año 2015, con 1 publicación, hasta 2018, con 4; aunque en los dos años posteriores, 2019 y 2020, no presenten producción sobre el tema.

Tabla 9. Distribución de documentos por fuente (top 5)

\begin{tabular}{|l|c|}
\hline \multicolumn{1}{|c|}{ Título de la fuente } & Documentos \\
\hline Information Communication And Society & 5 \\
\hline Media International Australia & 5 \\
\hline Australasian Journal Of Information Systems & 4 \\
\hline Health Promotion International & 3 \\
\hline Media And Communication & 2 \\
\hline
\end{tabular}

Fuente: Scopus

Gráfico 8. Documentos por año y fuente

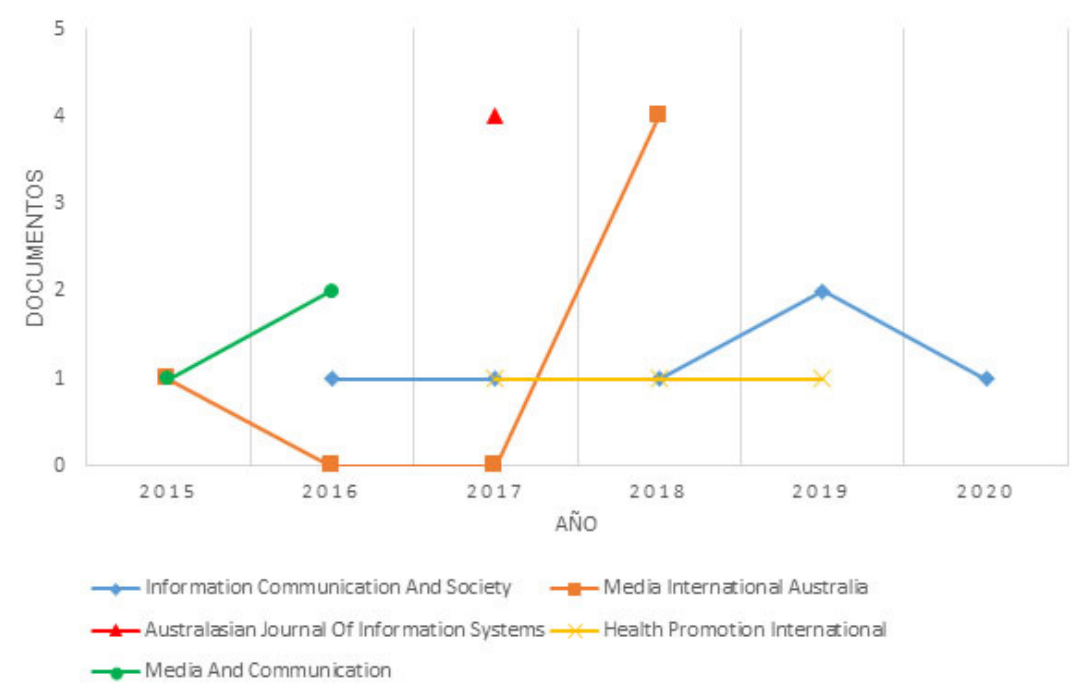

Fuente: Scopus 
Si limitamos los estadísticos descriptivos a solo los artículos científicos, tenemos 52 que abordan las implicancias de las narrativas producidas y reproducidas por indígenas para sus pueblos, a través de los nuevos medios. El análisis llega a valores importantes, los mismos que revelan aspectos útiles para los interesados en esta temática. Así, en la Tabla 10 encontramos que el valor promedio del número de citaciones hechas a los artículos científicos sobre el impacto de la participación de los indígenas en los medios sociales es de 4.56. El valor que más se repite entre estos documentos es o citas, que es a su vez es el valor mínimo alcanzado de citaciones; el máximo número de veces que un artículo fue citado es de 30 y ese documento es el realizado por Hanna, P., Langdon, E. J. y Vanclay, F. en 2016, titulado Indigenous rights, performativity and protest, que tiene un valor de 36.894 en su percentil de prominencia, y ha referenciado a 86 documentos que también se hallan en Scopus. Asimismo, los 52 artículos juntos fueron citados 237 veces.

Tabla 1o. Valores estadísticos del número de citas, percentil de prominencia alcanzado y cantidad de referencias realizadas de los artículos

\begin{tabular}{|l|l|l|l|}
\hline & N. $^{\circ}$ de citas & Percentil de prominencia & N. $^{\circ}$ de referencias \\
\hline Media & 4.56 & 75.02 & 47.37 \\
\hline Mediana & 2 & 84.07 & 44.50 \\
\hline Moda & 0 & 65.42 & 31 \\
\hline Mínimo & 0 & 17.56 & 0 \\
\hline Máximo & 30 & 99.94 & 95 \\
\hline Suma & 237 & 3900.81 & 2463 \\
\hline Cuenta & 52 & 52 & 52 \\
\hline
\end{tabular}

Fuente: Elaboración propia

Sobre el percentil de prominencia de cada artículo, según la misma tabla, el promedio alcanzado es de 75.02. EL valor que se encuentra en el medio de los percentiles alcanzados es 84.07, y el valor que más se repite es 65.42. El artículo Multicultural Feeling, Feminist Rage, Indigenous Refusal (Burman, 2016) es el que tiene el valor mínimo de percentil de prominencia, 17.56, mientras que el mayor es de 99.94, y se trata de An analysis of social media marketing of indigenous construction firms in Nigeria: A tool for sustainable growth (Adedeji, Rapheal, \& Opeyemi, 2018). 
Tabla 11. Top 10 de artículos científicos por percentil de prominencia alcanzado

\begin{tabular}{|c|c|c|c|c|c|}
\hline Título de documento & Autor & Año & Citas & Percentil & Referencias \\
\hline $\begin{array}{l}\text { Indigenous radio and digital } \\
\text { media: Tautoko FM's national and } \\
\text { transnational audiences }\end{array}$ & $\begin{array}{l}\text { de Bruin, J., } \\
\text { Mane, J. }\end{array}$ & 2018 & 2 & 99.941 & 39 \\
\hline $\begin{array}{l}\text { When the Personal Is Political: Ethnic } \\
\text { Identity, Ally Identity, and Political } \\
\text { Engagement Among Indigenous } \\
\text { People and People of Color }\end{array}$ & $\begin{array}{l}\text { Fish, J., Aguilera, } \\
\text { R., Ogbeide, I.E., } \\
\text { Ruzzicone, D.J., } \\
\text { Syed, M. }\end{array}$ & 2020 & 0 & 99.263 & 52 \\
\hline $\begin{array}{l}\text { Indigenous Movements, Collective } \\
\text { Action, and Social Media: New } \\
\text { Opportunities or New Threats? }\end{array}$ & Lupien, P. & 2020 & 1 & 98.348 & 39 \\
\hline $\begin{array}{l}\text { Nativist-populism, the internet and } \\
\text { the geopolitics of indigenous diaspora }\end{array}$ & Harris, J. & 2020 & 0 & 98.348 & 95 \\
\hline $\begin{array}{l}\text { Addressing the imagination gap } \\
\text { through STEAMM+D and indigenous } \\
\text { knowledge }\end{array}$ & Diamond, S. & 2019 & 2 & 97.858 & 63 \\
\hline $\begin{array}{l}\text { China's minority preferential policies } \\
\text { and the schooling of indigenous } \\
\text { Tibetan children: the weakest link }\end{array}$ & Phuntsog, N. & 2019 & 0 & 97.858 & 53 \\
\hline $\begin{array}{l}\text { Sacred sites protection and } \\
\text { indigenous women's activism: } \\
\text { Empowering grassroots social } \\
\text { movements to influence public policy. } \\
\text { a look into the "women of standing } \\
\text { rock" and "idle no more" indigenous } \\
\text { movements }\end{array}$ & Gottardi, F. & 2020 & 0 & 96.449 & 59 \\
\hline $\begin{array}{l}\text { Understanding the ways missing and } \\
\text { murdered Indigenous women are } \\
\text { framed and handled by social media } \\
\text { users }\end{array}$ & $\begin{array}{l}\text { Moeke-Pickering, } \\
\text { T., Cote-Meek, S., } \\
\text { Pegoraro, A. }\end{array}$ & 2018 & 0 & 95.535 & 46 \\
\hline $\begin{array}{l}\text { Canada is \#IdleNoMore: exploring } \\
\text { dynamics of Indigenous political and } \\
\text { civic protest in the Twitterverse }\end{array}$ & $\begin{array}{l}\text { Raynauld, } \\
\text { V., Richez, E., } \\
\text { Boudreau Morris, } \\
\text { K. }\end{array}$ & 2018 & 12 & 95.164 & 81 \\
\hline $\begin{array}{l}\text { Digital survivance and Trickster } \\
\text { humor: exploring visual and digital } \\
\text { Indigenous epistemologies in the } \\
\text { \# NoDAPL movement }\end{array}$ & $\begin{array}{l}\text { Hinzo, A.M., Clark, } \\
\text { L.S. }\end{array}$ & 2019 & 3 & 95.004 & 29 \\
\hline
\end{tabular}

Fuente: Scopus

De acuerdo al número de referencias hechas en cada artículo, tenemos que el promedio de todos los artículos juntos es de 47.37, el valor central es de 44.50 y el valor más repetido es 31 . Así también, 13 es cantidad mínima que un artículo ha referenciado documentos, y 95 el máximo. Cabe destacar que se halló entre los 52 artículos científicos solo uno que tiene como lengua original al castellano y es The digital attached in the indigenous youths: Between inequalities and local representations (Ramos-Mancilla, 2020), ingresado a Scopus este año. 


\section{Discusión}

Hasta la fecha, los interesados todavía no cuentan con una investigación documental que les sirva como referente o estado de arte en el que se apoyen para construir, rechazar y respaldar conclusiones respecto a temáticas relacionadas a la influencia de los indígenas y sobre lo indígena en los medios de comunicación social. La presente investigación es útil para la claridad en el establecimiento de qué es y qué no es "indígena" y cuál es la influencia que la etiqueta "indígena" va alcanzando en y a través de los nuevos medios, pues junto a la metodología del análisis bibliométrico, este trabajo también colabora objetivamente en el aspecto referencial y evolutivo de los "medios sociales indígenas" como agenda global (Ginsburg, 1993, 2016; Himpele, 2004), y en las perspectivas y aspiraciones de un mundo que mientras más converge mediáticamente es más multicultural.

La indigenous media y la misma cuestión "indígena" es un tópico progresista que se va liberando de populismos y otros sesgos. Esta investigación acude al análisis bibliométrico porque como metodología se desarrolla justamente, en este contexto, para superar esa y demás subjetividades (Ariza \& Quevedo, 2012; Wang \& Ngai, 2020). Se entiende que hay un peligro en encasillar todo progresismo a ideales utópicos, en creer que toda acción y política progresista es mala, en unidireccionar al progresismo como populismos de izquierda y negar que emergen populismos de derecha (Biglieri, 2020; Brito, 2020). El objetivo de analizar la producción científica reunida en la base de datos Scopus sobre la cuestión "indígena" en y a través de los medios sociales, ayuda a superar la idea de que multiculturalismo es populismo, y sobre todo a entender que el multicultarismo es también transmediático.

Puesto que los nuevos medios son la gran interfaz de la era de la convergencia, de la nueva ecología mediática, a la que acuden más y más personas en el mundo de distintas culturas, el intercambio cultural allí es altamente considerable, y uno de los factores más fundamentales para la creación y recreación de mundos narrativos. La interculturalidad está, más ahora que nunca, marcada por la convivencia del ser humano con los medios de comunicación; la cultura digital es en esencia intercultural y tiene como "meta-medio" al Internet, que contacta personas y símbolos procedentes de diversos contextos socioculturales (Rodríguez-Izquierdo, 2015; Noguera-Vivo, 2016). Por tanto, la investigación documental sobre la participación de lo indígena en los nuevos medios es un trabajo que brinda luces en torno al impacto de la ecología de los medios en el mundo y en sus pobladores, indígenas y no indígenas.

\section{Conclusiones}

Si bien la documentación que se reúne en Scopus sobre lo indígena en medios sociales no es copiosa en número, sí goza de muy buena calidad (el promedio de percentil de prominencia es de 75.2), y ello gracias a los trabajos realizados por 
científicos atentos al impacto sociopolítico de las campañas establecidas por los indígenas australianos que les permitieron acceder a espacios de influencia y de poder a través de los nuevo medios. El análisis bibliométrico indica que los 10 primeros artículos científicos con el mayor percentil de prominencia fijado por Scopus superan el valor de 95; es decir que, según el análisis de las citas y visitas, la producción científica relacionada a lo indígena en medios sociales alcanza un nivel de interés mayor al 95\%. Así también, se descubre que el interés de los investigadores por los tópicos que relacionan a los indígenas con la nueva ecología mediática ha crecido desde 2013 hasta la fecha (2020) de manera progresiva, pasando de 1 a 15 publicaciones por año sobre el tema.

Los datos ya sugieren implicancias interesantes del uso de los nuevos medios por los indígenas, y que estos se extienden más allá de lo social, pues alcanzan a su vez los campos de la salud, de lo psicológico, de la tecnología e ingeniería, las ciencias ambientales y demás terrenos y estudios científicos. Tal es el impacto de participación de lo indígena en los nuevos medios que el área de Negocios, Gestión y Contabilidad va sumando un 6,3\% del total de los 70 documentos relacionados al tema. Asimismo, ya se comienza a extender producción científica al respecto desde las áreas de Ciencias Agrícolas y Biológicas, Ciencias de la Tierra y del Planeta, Economía, Econometría y Finanzas, y Enfermería, las que hacen juntas 3,6\% de toda la documentación según área temática.

La documentación analizada devela la importancia de los medios de comunicación social en el desarrollo de las comunidades minoritarias, que perfilan una narrativa con identidad y en el caso indígena se trata de una narrativa con identidad indígena, que promueve elementos indígenas. Así, lo que acontece en Australia es el primer referente de ello, y luego Canadá. Por consiguiente, esta investigación bibliométrica concluye que la evidencia científica en Scopus sobre la influencia de lo indígena en y a través de los nuevos medios nos dice que hay una evolución positiva de este tema que radica en algo más que su visibilidad, puesto que va evolucionando también con respecto a su ubicuidad - la capacidad ciudadana de estar activa y creativamente presente en muchos lugares y espacios (Aparicio, 2013) - , y a su glocalidad - la capacidad de conectar lo que se habla de la vida local con lo que se habla a escala global (Garduño, 2016)—; esta es una manifestación de que en esta era convergente la cuestión indígena avanza del paternalismo hacía el empoderamiento.

\section{Referencias bibliográficas}

Adedeji, A., Rapheal, O., \& Opeyemi, O. (2018). An analysis of social media marketing of indigenous construction firms in nigeria: A tool for sustainable growth. International Journal of Construction Supply Chain Management, 8(2), 60-72. doi:10.14424/ijcscm802018-60-72

Aparicio González, D. (2013). Challenges and opportunities in documentary film: Ubiquity, collaboration and citizen participation. [Retos y oportunidades en el documental audiovisual: Ubicuidad, colaboración y participación ciudadana] Historia y Comunicacion Social, 18(SPEC. ISSUE NOV), 387-398. doi:10.5209/rev-HICS.2013.v18.44,250 
Ariñez Roca, Néstor Sebastián. (2007). Medios de comunicación social y conflictos. Punto Cero, 12(15), 08-15. Recuperado de http://www.scielo.org.bo/scielo.php? script=sci_arttext\&pid $=$ S1815-02762007000200002\&lng $=$ es\&tlng=es

Ariza, T., \& Quevedo-Blasco, R. (2012). Análisis bibliométrico de la Revista de Investigación Educativa (2000-2012). Revista de Investigación Educativa, 31(1), 31-52. https://doi. org/10.6018/rie.31.1.160321

Arruda, G. M., \& Krutkowski, S. (2017). Arctic governance, indigenous knowledge, science and technology in times of climate change: Self-realization, recognition, representativeness. Journal of Enterprising Communities, 11(4), 514-528. doi:10.1108/JEC-08-2015-0041

Banco Mundial. (s. f.). Pueblos indígenas: Panorama general. Recuperado de https://www. bancomundial.org/es/topic/indigenouspeoples

Berg-Nordlie, M. (2018). The governance of urban indigenous spaces: Norwegian sámi examples. Acta Borealia, 35(1), 49-72. doi:10.1080/08003831.2018.1457316

Biglieri, P. (2020). Populism, right-wing and left-wing position? [Populismo: ¿izquierdas y derechas?] Recerca, 25(1), 5-24. doi:10.6035/RECERCA.2020.25.1.2

Bhagwat, A., \& Jijina, P. (2020). A psychosocial lens on an indigenous initiative to address menstrual health and hygiene in indian villages. Social Work in Public Health, 35(3), 73-89. doi:10.1080/19371918.2020.1738972

BDPI. (s. f.). Lista de pueblos indígenas u originarios. Recuperado de https://bdpi.cultura. gob.pe/pueblos-indigenas

Brito, R. C. (2020). The issue of universality in the populist theory: In defense of a democratic populism. [El problema de la universalidad en la teoría populista: En defensa de un populismo democrático]. Recerca, 25(1), 47-69. doi:10.6035/RECERCA.2020.25.1.4

Brown, K., Toombs, M., Nasir, B., Kisely, S., Ranmuthugala, G., Brennan-Olsen, S. L., (...), \& Hides, L. (2020). How can mobile applications support suicide prevention gatekeepers in australian indigenous communities? Social Science and Medicine, 258 doi:10.1016/j. socscimed.2020.113015

Bruin, J. de, \& Mane, J. (2018). Indigenous radio and digital media: Tautoko FM's national and transnational audiences. Radio Journal, 16(2), 127-140. doi:10.1386/rjao.16.2.127_1

Burman, J. (2016). Multicultural feeling, feminist rage, indigenous refusal. Cultural StudiesCritical Methodologies, 16(4), 361-372. doi:10.1177/1532708616638693

Cárdenas, Cynthia Giovanna, Pesantes, María Amalia, \& Rodríguez, Alfredo. (2017). Interculturalidad en salud: reflexiones a partir de una experiencia indígena en la Amazonía peruana. Anthropologica, 35(39), 151-169. https://dx.doi.org/10.1880o/anthropologica.201702.007

Carlson, B., \& Dreher, T. (2018). Introduction: Indigenous innovation in social media. Media International Australia, 169(1), 16-20. doi:10.1177/1329878X18803798

Carlson, B., \& Frazer, R. (2020). "They got filters": Indigenous social media, the settler gaze, and a politics of hope. Social Media and Society, 6(2) doi:10.1177/2056305120925261

Carlson, B., Jones, L. V., Harris, M., Quezada, N., \& Frazer, R. (2017). Trauma, shared recognition and indigenous resistance on social media. Australasian Journal of Information Systems, 21 doi:10.3127/ajis.v21io.1570

Chamberlain, C., Perlen, S., Brennan, S., Rychetnik, L., Thomas, D., Maddox, R., (...), \& Eades, S. (2017). Evidence for a comprehensive approach to aboriginal tobacco control to maintain the decline in smoking: An overview of reviews among indigenous peoples. Systematic Reviews, 6(1) doi:10.1186/s13643-017-0520-9

Chávez, H. (2020). Calidad de las revistas científicas electrónicas de comunicación en Amé- 
rica Latina y España. (Tesis para optar el grado de Magíster en Comunicación Social con mención en Investigación en Comunicación. Unidad de Posgrado, Facultad de Letras y Ciencias Humanas, Universidad Nacional Mayor de San Marcos). Lima, Perú.

Cocq, C. (2015). Indigenous voices on the web: Folksonomies and endangered languages. Journal of American Folklore, 128(509), 273-285. doi:10.54,06/jamerfolk.128.509.0273

Corona, J. (2016). ¿Cuándo es transmedia?: discusiones sobre lo transmedia(l) de las narrativas. Icono $14.14(1)$, pp. 30-48.

Corona, S. B., \& Matta, R. (2020). Mestizo cuisines in Mexico and Peru. keys to interpreting multiculturalism in Latin America. [As culinárias mestiças no México e no Peru. Soluções para interpretar o multiculturalismo na América Latina; Las cocinas mestizas en México y Perú. Claves para interpretar el multiculturalismo en América Latina]. Antipoda, 2020(39), 69-93. doi:10.7440/antipoda39.2020.04.

Diamond, S. (2019). Addressing the imagination gap through STEAMM+D and indigenous knowledge. Proceedings of the National Academy of Sciences of the United States of America, 116(6), 1851-1856. doi:10.1073/pnas.1808679115

Dreher, T., McCallum, K., \& Waller, L. (2016). Indigenous voices and mediatized policy-making in the digital age. Information Communication and Society, 19(1), 23-39. doi:10.1080 /1369118X.2015.1093534

Du, J. T., \& Haines, J. (2017). Indigenous australians' information behaviour and internet use in everyday life: An exploratory study. Information Research, 22(1).

Duarte, M. (2017). Connected activism: Indigenous uses of social media for shaping political change. Australasian Journal of Information Systems, 21 doi:10.3127/ajis.v21io.1525

Fish, J., Aguilera, R., Ogbeide, I. E., Ruzzicone, D. J., \& Syed, M. (2020). When the personal is political: Ethnic identity, ally identity, and political engagement among indigenous people and people of color. Cultural Diversity and Ethnic Minority Psychology. doi:10.1037/ cdpoooo341

García Serrano, Fernando. (2014). Territorialidad y autonomía, proyectos minero-energéticos y consulta previa: el caso de los pueblos indígenas de la Amazonia ecuatoriana. Anthropologica, 32(32), 71-85. Recuperado de http://www.scielo.org.pe/scielo.php?script $=$ sci_arttext\&pid=So254-92122014000100004\&lng=es\&tlng=es

Garduño García, Moisés. (2016). Resonancias del zapatismo mexicano y la resistencia palestina: dos ejemplos de autonomía en el Sur Global. Espiral (Guadalajara), 23(65), 125-163. Recuperado de http://www.scielo.org.mx/scielo.php?script=sci_arttext\&pi$\mathrm{d}=\mathrm{S} 1665-05652016000100004 \& \operatorname{lng}=$ es\&tlng=es

Gendron, F., Hancherow, A., \& Norton, A. (2017). Exploring and revitalizing indigenous food networks in saskatchewan, canada, as a way to improve food security. Health Promotion International, 32(5), 808-817. doi:10.1093/heapro/dawo13

Gestión, Noticias. (2019, noviembre 12). Mundo: Los países con la mayor cantidad de población indígena de América | Noticias Gestión Perú. Gestión. https://gestion.pe/mundo/ los-paises-con-la-mayor-cantidad-de-poblacion-indigena-de-america-noticia/

Ginsburg, Faye. (2016). Indigenous media from u-matic to Youtube: Media sovereignty in the digital age. Sociologia \& Antropologia, 6(3), 581-599. https://doi.org/10.159o/2238$38752016 v 632$

Gottardi, F. (2020). Sacred sites protection and indigenous women's activism: Empowering grassroots social movements to influence public policy. A look into the "women of standing rock" and "idle no more" indigenous movements. Religions, 11(8), 1-13. doi:10.339o/ rel11080380 
Graham, L. R. (2016). Toward representational sovereignty: Rewards and challenges of indigenous media in the A'uw -xavante communities of eténhiritipa-pimentel barbosa. Media and Communication, 4(2), 13-32. doi:10.17645/mac.v4i2.438

Grandia, L. (2020). Back to the future: The autonomous indigenous communities of petén, guatemala. [De volta para o futuro: As comunidades indígenas autônomas de Petén, Guatemala; Retorno al futuro: Las comunidades indígenas autónomas de Petén, Guatemala]. Antipoda, 2020(40), 103-127. doi:10.7440/antipoda40.2020.05

Hanna, P., Langdon, E. J., \& Vanclay, F. (2016). Indigenous rights, performativity and protest. Land use Policy, 50, 490-506. doi:10.1016/j.landusepol.2015.06.034

Harris, J. (2020). Nativist-populism, the internet and the geopolitics of indigenous diaspora. Political Geography, 78 doi:10.1016/j.polgeo.2019.102124

Hefler, M., Kerrigan, V., Henryks, J., Freeman, B., \& Thomas, D. P. (2019). Social media and health information sharing among australian indigenous people. Health Promotion International, 34(4), 706-715. doi:10.1093/heapro/dayo18

Hernández-González, V, Sans-Rosell, N, Jové-Deltell, M. C, \& Reverter-Masia, J. (2016). Comparación entre Web of Science y Scopus, Estudio Bibliométrico de las Revistas de Anatomía y Morfología. International Journal of Morphology, 34(4), 1369-1377. https:// dx.doi.org/10.4067/So717-95022016000400032

Hinzo, A. M., \& Clark, L. S. (2019). Digital survivance and trickster humor: Exploring visual and digital indigenous epistemologies in the \#NoDAPL movement. Information Communication and Society, 22(6), 791-807. doi:10.1080/1369118X.2019.1573911

Himpele, J. (2004). Packaging Indigenous Media: An Interview with Ivan Sanjinés and Jesús Tapia. American Anthropologist, 106(2), 354-363. doi:10.1525/aa.2004.106.2.354

Hutchings, S., \& Rodger, D. (2018). Reclaiming Australia: Indigenous hip-hop group A.B. Original's use of twitter. Media International Australia, 169(1), 84-93. doi:10.1177/1329878X18803382

Junka-Aikio, L. (2018). Indigenous culture jamming: Suohpanterror and the articulation of sami political community. Journal of Aesthetics and Culture, 10(4) doi:10.1080/200 04.214.2017.1379849

Jenkins, H., Ford, S., \& Green, J. [2013] (2015). Cultura transmedia. La creación de contenido y valor en una cultura en red. (Xavier Gaillard, trad.). Madrid: Gedisa.

Jenkins, H. [2006] (2008). Convergence Culture. La cultura de la convergencia de los medios de comunicación. (Pablo Hermida, trad.). Madrid: Paidós.

Kidd, D. (2020). Standing rock and the indigenous commons. Popular Communication, 1-15. doi:10.1080/15405702.2020.1781862

Kira, A., Glover, M., Walker, N., \& Bauld, L. (2016). Recruiting pregnant indigenous women who smoke into a high contact incentivized cessation trial: A feasibility study. Nicotine and Tobacco Research, 18(10), 2036-2040. doi:10.1093/ntr/ntw106

Knezevic, I., Pasho, J., \& Dobson, K. (2018). Seal hunts in canada and on twitter: Exploring the tensions between indigenous rights and animal rights with \#sealfie. Canadian Journal of Communication, 43(3), 421-439. doi:10.2223o/cjc.2017v43n3a3376

Kortelainen, T., \& Länsman, A. (2015). Challenging the status and constructing the culture of an indigenous group: Attention paid to YLE Sápmi radio digital news in Finland. European Journal of Cultural Studies, 18(6), 690-708. doi:10.1177/1367549415572326

Kral, I. (2014). Shifting perceptions, shifting identities: Communication technologies and the altered social, cultural and linguistic ecology in a remote indigenous context. Australian Journal of Anthropology (the), 25(2), 171-189. doi:10.1111/taja.12087 
Latimore, J., Nolan, D., Simons, M., \& Khan, E. (2017). Reassembling the indigenous public sphere. Australasian Journal of Information Systems, 21 doi:10.3127/ajis.v21io.1529

Leonardi, Paul M., Huysman, Marleen, \& Steinfield, Charles. (2013). Enterprise Social Media: Definition, History, and Prospects for the Study of Social Technologies in Organizations. Journal of Computer-Mediated Communication, Vol. 19, Issue 1, 1 October 2013, 1-19. https://doi.org/10.1111/jec4.12029

Louie, D. W. (2017). Social media and the sexual exploitation of indigenous girls. Girlhood Studies, 10(2), 97-113. doi:10.3167/ghs.2017.100208

Luna Habito de, C., \& Ealdama, S. J. G. (2019). Identity construction, social media, and ifugao rice terraces conservation of indigenous people's youth through appreciative inquiry. International Journal of Social Ecology and Sustainable Development, 10(4), 47-59. doi:10.4018/IJSESD.2019100104

Lupien, P. (2020). Indigenous movements, collective action, and social media: New opportunities or new threats? Social Media and Society, 6(2) doi:10.1177/2056305120926487

Lupien, P., \& Chiriboga, G. (2019). Use of information and communications technologies by indigenous civil society organizations in Ecuador. Information Communication and Society, 22(8), 1029-1043. doi:10.1080/1369118X.2017.1397182

McCallum, K., Waller, L., \& Dreher, T. (2016). Mediatisation, marginalisation and disruption in australian indigenous affairs. Media and Communication, 4(4), 30-42. doi:10.17645/ mac.v4i4.695

McLean, S., Wilson, A., \& Lee, E. (2017). The whiteness of redmen: Indigenous mascots, social media and an antiracist intervention. Australasian Journal of Information Systems, 21 doi:10.3127/ajis.v21io.1590

McPhail-Bell, K., Appo, N., Haymes, A., Bond, C., Brough, M., \& Fredericks, B. (2018). Deadly choices empowering indigenous australians through social networking sites. Health Promotion International, 33(5), 770-780. doi:10.1093/heapro/daxo14.

Miner, J. D. (2020). Informatic tactics: Indigenous activism and digital cartographies of gender-based violence. Information Communication and Society, doi:10.1080/136911 8X.2020.1797851

Moeke-Pickering, T., Cote-Meek, S., \& Pegoraro, A. (2018). Understanding the ways missing and murdered indigenous women are framed and handled by social media users. Media International Australia, 169(1), 54-64. doi:10.1177/1329878X18803730

OIT. (2014). Convenio N. ${ }^{\circ} 169$ de la OIT sobre Pueblos Indígenas y Tribales en países independientes. Declaración de las Naciones Unidas sobre los Derechos de los Pueblos Indígenas. Lima: OIT / Oficina Regional para América Latina y el Caribe.

ONU. (s.f.). Los Pueblos Indígenas en las Naciones Unidas. Recuperado de https://www. un.org/development/desa/indigenous-peoples-es/historia.html

Owiny, S. A., Mehta, K., \& Maretzki, A. N. (2014). The use of social media technologies to create, preserve, and disseminate indigenous knowledge and skills to communities in east Africa. International Journal of Communication, 8(1), 234-247.

Pérez-Van-Leenden, M. (2019). La investigación acción en la práctica docente. Un análisis bibliométrico (2003-2017). Magis, Revista Internacional de Investigación en Educación, 12 (24), 177-192. doi: 10.11144/Javeriana.m10-20.ncev

Phuntsog, N. (2019). China's minority preferential policies and the schooling of indigenous tibetan children: The weakest link. Intercultural Education, 30(1), 68-82. doi:10.1080/1 4675986.2018.1534040

Ramos-Mancilla, O. (2020). The digital attached in the indigenous youths: Between inequal- 
ities and local representations. [O agregado digital em jovens indígenas: Entre desigualdades e representações locais; El agregado digital en las juventudes indígenas: Entre desigualdades y representaciones locales]. Perspectivas em Ciencia da Informacao, 25(1), 263-282. doi:10.1590/1981-5344/3870

Raynauld, V., Richez, E., \& Boudreau Morris, K. (2018). Canada is \#IdleNoMore: Exploring dynamics of indigenous political and civic protest in the twitterverse. Information Communication and Society, 21(4), 626-642. doi:10.1080/1369118X.2017.1301522

Real Academia Española. (2001). Diccionario de la lengua española (23.a ed.). Madrid: RAE.

Riorda, M. (2017). Redes sociales para gobernar. Una mirada de América Latina. Nueva Sociedad. 269(1), 89-95.

Rosado-May, Francisco J. (2017). Formación universitaria intercultural para indígenas mayas de Yucatán, México. Anthropologica, 35(39), 215-242. https://dx.doi.org/10.188oo/ anthropologica.201702.009

Ryan, T. (2016). Seen but unseen: Missing visible indigenous women in the media and what it means for leadership in indigenous Australia. Platform, 7(Specialissue), 26-34

Scolari, C. (ed.) (2018). Adolescentes, medios de comunicación y culturas colaborativas: aprovechando las competencias transmedia de los jóvenes en el aula. Barcelona: Transmedia Literacy H2020 Research and Innovation Action / Universitat Pompeu Fabra.

Scolari, C. (2013). Narrativas Transmedia. Cuando todos los medios cuentan. Barcelona: Deusto.

Thomas Poell (2014) Social media and the transformation of activist communication: exploring the social media ecology of the 2010 Toronto G2O protests. Information, Communication \& Society, 17:6, 716-731. doi:10.1080/1369118X.2013.812674

Titifanue, J., Varea, R. R., Varea, R., Kant, R., \& Finau, G. (2018). Digital diaspora, reinvigorating indigenous identity and online activism: Social media and the reorientation of rotuman identity. Media International Australia, 169(1), 32-42. doi:10.1177/1329878X18803377

Trees, K. (2015). Mobile media: Communicating with and by indigenous youth about alcohol. Australian Aboriginal Studies, (1), 97-106.

Tsai, J. Y., Bosse, R., Sridharan, N., \& Chadha, M. (2020). Reclaiming the narratives: Situated multidimensional representation of underserved Indigenous communities through citizen-driven reporting. Journalism. https://doi.org/10.1177/1464884920983261

Universitat Politècnica de València. (27 de mayo de 2015). Revisión Sistemática de literatura. Módulo 1. Revisiones sistemáticas frente a meta-análisis. [Vídeo]. Youtube. https://www. youtube.com/watch? $\mathrm{v}=\mathrm{xsci} \_$liatzQ

UNDP. (s.f.). Los Pueblos Indígenas en el Perú. Recuperado de https://www.pe.undp.org/content/peru/es/home/presscenter/articles/2019/los-pueblos-indigenas-en-el-peru.html

Unesco. (2017, septiembre 25). Pueblos indígenas. Recuperado de https://es.unesco.org/ indigenous-peoples; https://plus.google.com/+UNESCO

Vicente Torrico, David. (2017). Estudio bibliométrico de la producción científica sobre narrativa transmedia en España hasta 2016: Análisis descriptivo de las 20 principales revistas de comunicación españolas según Google Scholar Metrics (h5). En adComunica. Revista Científica de Estrategias, Tendencias e Innovación en Comunicación, N. ${ }^{\circ} 14$. Castellón: Asociación para el Desarrollo de la Comunicación adComunica y Universitat Jaume I, 141-160. doi:http://dx.doi. org/10.6035/2174-0992.2017.14.8

Walker, T., Molenaar, A., \& Palermo, C. (2020). A qualitative study exploring what it means to be healthy for young indigenous australians and the role of social media in influencing health behaviour. Health Promotion Journal of Australia, doi:10.1002/hpja.391 
Waller, L., Dreher, T., \& McCallum, K. (2015). The listening key: Unlocking the democratic potential of indigenous participatory media. Media International Australia, (154), 57-66. doi:10.1177/1329878x1515400109

Wang, Q., \& Ngai, E.W.T. (2020). Event study methodology in business research: a bibliometric analysis. Industrial Management \& Data Systems, Vol. 120, N. ${ }^{\circ}$ 10, 1863-1900. https:// doi.org/10.1108/IMDS-12-2019-0671

Xuan Zhao, Cliff Lampe, \& Nicole B. Ellison. (2016). The Social Media Ecology: User Perceptions, Strategies and Challenges. Proceedings of the 2016 CHI Conference on Human Factors in Computing Systems. Association for Computing Machinery, New York, NY, USA, 89-100. DOI:https://doi.org/10.1145/2858036.2858333 